\title{
Biomarkers related to severe hypoglycaemia and lack of good glycaemic control in ACCORD
}

\author{
Lisa S. Chow • Haiying Chen • Michael E. Miller • \\ Santica M. Marcovina $\cdot$ Elizabeth R. Seaquist
}

Received: 19 November 2014 / Accepted: 9 January 2015 / Published online: 5 February 2015

(C) Springer-Verlag Berlin Heidelberg 2015

\begin{abstract}
Aims/hypothesis In patients with diabetes, intensive glycaemic control reduces microvascular complications. However, severe hypoglycaemia frequently complicates intensive glycaemic control. Blood biomarkers that predict successful intensification of glycaemic control in patients with type 2 diabetes without the development of severe hypoglycaemia would advance patient care. In patients who received intensive treatment for type 2 diabetes from the Action to Control Cardiovascular Risk in Diabetes (ACCORD) study, we hypothesised that insulin deficiency and islet autoantibodies would be associated with severe hypoglycaemia and failure to achieve near-normal glycaemia $\left(\mathrm{HbA}_{1 \mathrm{c}}<6.0 \%\right.$ [42 $\mathrm{mmol} / \mathrm{mol}]$ ).

Methods A nested case-control design was used. Cases ( $n=$ 326) were defined as participants having severe hypoglycaemia and failure to achieve an $\mathrm{HbA}_{1 \mathrm{c}}$ level of $<6.0 \%$ (42 $\mathrm{mmol} / \mathrm{mol})$ prior to the ACCORD transition or death. Controls $(n=1,075)$ were those who achieved an $\mathrm{HbA}_{1 \mathrm{c}}$ level of $<6.0 \%(42 \mathrm{mmol} / \mathrm{mol})$ prior to the ACCORD transition or death without severe hypoglycaemia. Each case was matched (for race, age and BMI) by up to four controls. Baseline insulin deficiency (fasting C-peptide $\leq 0.15 \mathrm{nmol} / \mathrm{l}$ ) and islet autoantibodies
\end{abstract}

L. S. Chow $(\varangle) \cdot$ E. R. Seaquist

Department of Medicine, University of Minnesota, 420 Delaware St

SE, MMC 101, Minneapolis, MN 55455, USA

e-mail: chow0007@umn.edu

H. Chen • M. E. Miller

Department of Biostatistical Sciences, Wake Forest School of

Medicine, Winston-Salem, NC, USA

S. M. Marcovina

Department of Medicine, University of Washington,

Seattle, WA, USA (glutamic acid decarboxylase [GAD], tyrosine phosphatase-related islet antigen 2 [IA2], insulin [IAA] and zinc transporter 8 [ZnT8]) were measured. Conditional logistic regression with and without adjustment for age, BMI and diabetes duration was used on the full cohort and after removal of patients who died and their respective controls.

Results Severe hypoglycaemia accompanied by an inability to achieve an $\mathrm{HbA}_{1 \mathrm{c}}$ level of $<6.0 \%(42 \mathrm{mmol} / \mathrm{mol})$ was associated with insulin deficiency (adjusted OR 23.2 [95\% CI 9.0, 59.5], $p<0.0001$ ), the presence of IAA autoantibodies or baseline insulin use (adjusted OR 3.8 [95\% CI 2.7, 5.3], $p<0.0001$ ), GAD autoantibodies (OR 3.9 [95\% CI 2.5, 6.0], $p<0.0001$ ), IA2 autoantibodies (OR 16.7 [95\% CI 3.9, 71.6], $p=0.0001$ ) and ZnT8 autoantibodies (adjusted OR $3.9[95 \%$ CI 1.2, 12.4], $p=0.02$ ).

Conclusions C-peptide and islet autoantibodies may serve as biomarkers to predict the risk of severe hypoglycaemia during intensification of type 2 diabetes treatment.

Trial registration: ClinicalTrials.gov NCT00000620 (original ACCORD study)

Keywords ACCORD study $\cdot$ Insulin deficiency $\cdot$ Islet autoantibodies

$\begin{array}{ll}\text { Abbreviations } \\ \text { ACCORD } & \begin{array}{l}\text { Action to Control Cardiovascular Risk in } \\ \text { Diabetes }\end{array} \\ \text { DASP } & \begin{array}{l}\text { Diabetes Antibodies Standardisation Program } \\ \text { DK units }\end{array} \\ \text { Digestive and kidney units } \\ \text { GAD } & \text { Glutamic acid decarboxylase } \\ \text { IA2 } & \text { Tyrosine phosphatase-related islet antigen-2 } \\ \text { IAA } & \text { Insulin autoantibodies } \\ \text { LADA } & \text { Latent autoimmune diabetes of adults } \\ \text { NIDDK } & \text { National Institute of Diabetes and Digestive and } \\ & \text { Kidney Diseases }\end{array}$

Abbreviations

ACCORD Action to Control Cardiovascular Risk in

DASP Diabetes Antibodies Standardisation Program

DK units Digestive and kidney units

GAD Glutamic acid decarboxylase

IA2 Tyrosine phosphatase-related islet antigen-2

IAA Insulin autoantibodies

LADA Latent autoimmune diabetes of adults

Kidney Diseases 
NWRL Northwest Research Laboratories

SH Severe hypoglycaemia

ZnT8 Zinc transporter 8

\section{Introduction}

In patients with type 2 diabetes, severe hypoglycaemia (SH), defined as hypoglycaemia requiring assistance [1], is a serious complication of diabetes treatment. SH is increasingly recognised as a significant public health issue. It is associated with a high frequency of emergency room visits [2], major macrovascular events (death from a cardiovascular cause, non-fatal myocardial infarction or non-fatal stroke), major microvascular events (new or worsening nephropathy or retinopathy), cardiovascular mortality and total mortality [3].

In type 2 diabetes, $\mathrm{SH}$ frequently arises from intensive treatment strategies (typically involving insulin) to achieve near-normal glycaemia for reducing microvascular complications [3-5]. However, $\mathrm{SH}$ is not limited to patients with nearnormal $\mathrm{HbA}_{1 \mathrm{c}}$ levels $[6,7]$. $\mathrm{SH}$ in the setting of higher $\mathrm{HbA}_{1 \mathrm{c}}$ values may be more detrimental than $\mathrm{SH}$ in the setting of nearnormal $\mathrm{HbA}_{1 \mathrm{c}}$ values because of the effect of glucose variability on outcomes [8]. Therefore, the identification of patients with type 2 diabetes at risk of the development of SH during intensive glycaemic treatment is needed.

To identify potential blood biomarkers which predict the development of SH during type 2 diabetes treatment intensification, we analysed data from the Action to Control Cardiovascular Risk in Diabetes (ACCORD) study (ClinicalTrials.gov NCT00000620). Follow-up of ACCORD participants with clinically diagnosed type 2 diabetes randomised to intensive glycaemic treatment provides a prime opportunity to identify tolerance of treatment intensification relative to the achievement of glycaemic goals. We hypothesised that among ACCORD participants randomised to intensive glycaemic treatment, those who failed to achieve target glycaemia and experienced SH will show more evidence of insulin deficiency and/or islet cell autoantibodies than those who successfully achieved target glycaemia without experiencing $\mathrm{SH}$.

\section{Methods}

Participants The ACCORD study was a randomised, multicentre, double, $2 \times 2$ factorial design study examining the effect of glycaemic control (intensive vs standard), blood pressure control and lipid control on cardiovascular morbidity and mortality in patients with clinically diagnosed type 2 diabetes [9]. The study enrolled 10,251 participants with long- standing type 2 diabetes and either diagnosed cardiovascular disease or at least two cardiovascular risk factors (in addition to diabetes) for intervention and subsequent follow-up. All participants were randomised to either standard glycaemic treatment (target $\mathrm{HbA}_{1 \mathrm{c}}$ level 7.0-7.9\% [53-63 mmol/mol]) or intensive glycaemic treatment (target $\mathrm{HbA}_{1 \mathrm{c}}$ level $<6.0 \%$ [42 $\mathrm{mmol} / \mathrm{mol}]$ ). Partway through the study (median followup 3.7 years), participants receiving intensive treatment were transitioned to standard treatment because of the higher total and cardiovascular mortality in the intensive treatment group than in the standard treatment group. Hypoglycaemia which required assistance and either glucose levels of $<2.8 \mathrm{mmol} / 1$ or symptoms that promptly resolved with treatment was frequent in the ACCORD study, with an annual incidence of 5.05 events/100 person-years in the intensive group and 1.51 events/100 person-years in the standard group [5].

Study design Using only ACCORD participants who received intensive treatment, we re-analysed the data using a nested case-control design to examine whether baseline insulin deficiency and/or the presence of islet autoantibodies was associated with SH and an inability to achieve good glycaemic control. A case was defined as a participant who had at least one episode of SH during the ACCORD study and who also failed to achieve a target $\mathrm{HbA}_{1 \mathrm{c}}$ level of $<6.0 \%(42 \mathrm{mmol} / \mathrm{mol})$ at any point during the follow-up period. Failure to achieve this target was assessed by inspection of the quarterly levels of $\mathrm{HbA}_{1 \mathrm{c}}$ obtained from the ACCORD central laboratory. Since we were interested in the implications of SH affecting glycaemic control, our outcome focused on the inability to achieve a near-normal $\mathrm{HbA}_{1 \mathrm{c}}$ level given a history of $\mathrm{SH}$ and not the presence of $\mathrm{SH}$ alone. A control was defined as an ACCORD participant who achieved a target $\mathrm{HbA}_{1 \mathrm{c}}$ level of $<6.0 \%$ (42 $\mathrm{mmol} / \mathrm{mol}$ ) at any point during the follow-up period without $\mathrm{SH}$ and who was also at risk at follow-up of the $\mathrm{SH}$ event of the case. Each case was matched to four controls (exact match for race and optimal distance matching for age and BMI). Analyses of fasting C-peptide and islet autoantibodies were performed at baseline. Computerised matching of cases to controls was achieved using the DIST [10] and VMATCH [11] macros [12]. In total, 326 cases and 1,075 controls were selected. The study was considered exempt by the University of Minnesota Institutional Review Board and was approved by the ACCORD Presentation and Publication Committee. Study participants gave informed consent for their participation in the ACCORD study and any approved substudies by the ACCORD Presentation and Publication Committee.

ACCORD criteria for type 2 diabetes Participants were clinically screened for eligibility by reviewing their medical history and a physical examination. Participants enrolled in the ACCORD study were required to have a clinical diagnosis of 
type 2 diabetes, as defined by the 1997 ADA criteria, and to have undergone a stable diabetes treatment programme for at least 3 months. Participants with self-reported or previously diagnosed type 1 diabetes, secondary causes of diabetes, or gestational diabetes were excluded. C-peptide and islet autoantibody levels were measured from baseline samples after completion of the ACCORD study and were not used to determine initial ACCORD study eligibility.

C-peptide level as a surrogate for insulin deficiency Insulin deficiency was approximated by fasting C-peptide levels. Measurements of C-peptide were performed using an immunoenzymometric assay on a Tosoh AIA 1800 analyser (Tokyo, Japan), with intra- and interassay coefficients of variation of $<2.0 \%$ and $<5.0 \%$, respectively. An assay sensitivity limit of $0.02 \mathrm{nmol} / 1$ was established in the ACCORD central laboratory as part of the method validation and was verified for each reagent lot. We used fasting C-peptide levels to categorise participants as either insulin sufficient (C-peptide $>0.15 \mathrm{nmol} / \mathrm{l}$ ) or insulin deficient (C-peptide $\leq 0.15 \mathrm{nmol} / \mathrm{l}$ ), consistent with the literature $[13,14]$. Participants being treated with insulin at baseline were also classified into insufficient and insulin-deficient categories according to the C-peptide criteria. Table 1 summarises the classification criteria.

Islet autoantibody measurement Measured islet autoantibodies included those against glutamic acid decarboxylase (GAD), tyrosine phosphatase-related islet antigen-2 (IA2), insulin (IAA) and zinc transporter 8 (ZnT8). Analyses of GAD and IA2 were performed on baseline samples at the Northwest Research Laboratories (NWRL), University of Washington, a central laboratory of the ACCORD study. The NWRL is one of the laboratories in which the National Institute of Diabetes and Digestive and Kidney Diseases (NIDDK) standardised

Table 1 Measured blood biomarkers

\footnotetext{
${ }^{\text {a }}$ Normal range $0.17-1.0 \mathrm{nmol} / 1$

${ }^{\mathrm{b}}$ Also designated positive if baseline insulin use
}

procedure for detecting GAD and IA2 autoantibodies has been implemented. The procedure was developed in co-operation with the NIDDK Harmonization Programme and the Diabetes Antibodies Standardisation Program (DASP). In the most recent DASP workshop, the sensitivity and specificity of the GAD assay were $82 \%$ and $93.3 \%$, respectively. The sensitivity and specificity of the IA2 assay were $62 \%$ and $100 \%$, respectively. For the GAD assay, participants with $\geq 33$ digestive and kidney (DK) units/ml were considered GAD positive and those with $<33 \mathrm{DK}$ units $/ \mathrm{ml}$ were considered GAD negative. For the IA2 assay, participants with $\geq 5$ DK units $/ \mathrm{ml}$ were considered IA2 positive and $<5 \mathrm{DK}$ units $/ \mathrm{ml}$ were considered IA2 negative [15].

ZnT8 and IAA analyses were performed at the Barbara Davis Center, University of Colorado. ZnT8 results are expressed as an index. Based on a receiver operating characteristic curve for 100 controls and 50 patients with recentonset diabetes, a cutoff at a 0.02 index was determined. In the most recent DASP workshop (2010), the ZnT8 assay showed a specificity and sensitivity of $100 \%$ and $64 \%$, respectively. Similarly, IAA analysis results are expressed as an index, and a 0.01 index has been determined as the cutoff for controls. In the most recent DASP workshop, the IAA assay showed a specificity and sensitivity of $56 \%$ and $99 \%$, respectively. For ZnT8, participants with a $>0.02$ index were considered ZnT8 positive and those with a $\leq 0.02$ index were considered ZnT8 negative. For IAA, participants with either a $>0.01$ index or baseline insulin use were considered IAA positive, and those with $a \leq 0.01$ index were considered IAA negative. As the use of exogenous insulin may be associated with the formation of IAA [16], we made the conservative assumption that any exogenous insulin use will be associated with IAA positivity. Cutoff criteria are summarised in Table 1.

Statistical analysis We used a nested case-control design with the matched sets defining strata. Baseline variables used for matching were summarised using descriptive statistics for both cases and controls. Other baseline characteristics were compared between cases and controls using MantelHaenszel test statistics to adjust for correlations within strata. For each baseline risk factor (presence of insulin deficiency and islet autoantibodies), we fitted separate conditional logistic regression models to examine its association with case or control status (Model 1). Since age and BMI were matched as continuous variables, we also fitted multivariable conditional logistic regression models specifically adjusting for age, BMI and diabetes duration (Model 2). The full analysis was repeated by removing cases in which the patient died $(n=21)$ and their respective controls $(n=86$; Model 3 ). A similar set of analyses was conducted for the number of autoantibodies (categorised as 0,1 and $\geq 2$ ). The magnitude of associations between baseline risk factors and our primary outcome were quantified by ORs and their associated $p$ values. Finally, we 
conducted a sensitivity analysis using only those case-control pairs which matched within 5 years for age and $5 \mathrm{~kg} / \mathrm{m}^{2}$ for BMI. All analyses were performed using SAS version 9.3 (Cary, NC, USA) and a $p$ value of $<0.05$ was considered statistically significant.

\section{Results}

Characteristics of cases and controls The characteristics of cases and controls are shown in Table 2. Compared with controls, cases had higher $\mathrm{HbA}_{1 \mathrm{c}}$ levels, a longer duration of diabetes, a higher frequency of insulin deficiency (17\% of cases vs $1 \%$ of controls), and a higher percentage of insulin use ( $89 \%$ of cases vs $24 \%$ of controls) at baseline. Cases had comparable fasting glucose levels to controls, but higher rates of positive islet antibodies (GAD, IA2, IAA, ZnT8). The quality of the optimal matching on the continuous age and BMI variables was excellent: $90.9 \%$ of age matches were within 5 years, with a median difference of 0.5 years. For BMI, $90.7 \%$ of matches were within $5 \mathrm{~kg} / \mathrm{m}^{2}$, with a median difference of $1.21 \mathrm{~kg} / \mathrm{m}^{2}$.

Table 2 Baseline characteristics of cases and controls

\begin{tabular}{|c|c|c|c|}
\hline Variable & $\begin{array}{l}\text { Cases } \\
(n=326)\end{array}$ & $\begin{array}{l}\text { Controls } \\
(n=1,075)\end{array}$ & $p$ value \\
\hline Age (years) & $63.3(7.2)$ & $62.6(6.3)$ & Matched \\
\hline Sex (male) & $164(50 \%)$ & $639(59 \%)$ & 0.04 \\
\hline Race (white) & $183(56 \%)$ & $726(68 \%)$ & Matched \\
\hline BMI $\left(\mathrm{kg} / \mathrm{m}^{2}\right)$ & $31.6(5.5)$ & $32.5(5.2)$ & Matched \\
\hline $\mathrm{HbA}_{1 \mathrm{c}}(\%)$ & $8.5(1.0)$ & $8.1(1.0)$ & $<0.0001$ \\
\hline $\mathrm{HbA}_{1 \mathrm{c}}(\mathrm{mmol} / \mathrm{mol})$ & $69(10.9)$ & $65(10.9)$ & $<0.0001$ \\
\hline Fasting glucose (mmol/l) & $9.4(4.1)$ & $9.8(2.7)$ & 0.15 \\
\hline Duration of diabetes (years) & $15.1(8.9)$ & $9.3(6.9)$ & $<0.0001$ \\
\hline Insulin use at baseline & $191(89 \%)$ & $259(24 \%)$ & $<0.0001$ \\
\hline Insulin deficiency $^{\mathrm{a}}$ & $55(17 \%)$ & $8(1 \%)$ & $<0.0001$ \\
\hline GAD positive & $56(17 \%)$ & $62(6 \%)$ & $<0.0001$ \\
\hline IA2 positive & $12(4 \%)$ & $4(0.4 \%)$ & $<0.0001$ \\
\hline IAA positive or baseline insulin use & $202(62 \%)$ & $276(26 \%)$ & $<0.0001$ \\
\hline ZnT8 positive & $7(2 \%)$ & $7(0.7 \%)$ & 0.005 \\
\hline \multicolumn{4}{|l|}{ Number of positive autoantibodies } \\
\hline 0 & $108(33 \%)$ & $757(70 \%)$ & $<0.0001$ \\
\hline 1 & $174(53 \%)$ & $292(27 \%)$ & \\
\hline 2 & $33(10 \%)$ & $22(2 \%)$ & \\
\hline 3 & $7(2 \%)$ & $3(0.3 \%)$ & \\
\hline 4 & $4(1 \%)$ & $1(0.1 \%)$ & \\
\hline
\end{tabular}

Data are $n(\%)$ for categorical variables and mean (SD) for continuous variables

${ }^{\mathrm{a}} \mathrm{C}$-peptide $\leq 0.15 \mathrm{nmol} / 1$
Islet autoantibody profile of participants with baseline insulin deficiency All participants with insulin deficiency $(n=63)$ were taking insulin and therefore designated as IAA positive. Excluding IAA, the most common positive islet autoantibody was GAD (44.4\% in participants with insulin deficiency, $6.7 \%$ in those without insulin sufficiency). These findings persisted after the removal of cases involving patients who died $(n=21)$ and their respective controls $(n=86)$.

Odds of SH and failure to achieve optimal glycaemic control for participants with baseline insulin deficiency or islet autoantibodies Baseline insulin deficiency was associated with a higher OR (reported as OR [95\% CI]) of SH and failure to achieve an $\mathrm{HbA}_{1 \mathrm{c}}$ level of $<6.0(42 \mathrm{mmol} / \mathrm{mol})$ which persisted after adjusting for age, BMI, diabetes duration and exclusion of those who died during the study (unadjusted OR 35.6 [95\% CI 14.2, 89.6], $p<0.0001$; fully adjusted and after removing deaths, OR 23.2 [95\% CI 9.0, 59.5], $p<0.0001$ ). This outcome was also associated with a positive IAA and baseline insulin use (unadjusted OR 4.5 [95\% CI 3.4, 6.0], $p<0.0001$; fully adjusted and removing deaths, OR 3.8 [95\% CI 2.7, 5.3], $p<0.0001$ ), positive GAD (unadjusted OR 3.6 [95\% CI 2.4, 5.4], $p<0.0001$; fully adjusted and removing deaths, OR 3.9 [95\% CI 2.5, 6.0], $p<0.0001$ ), positive IA2 (unadjusted OR 10.6 [95\% CI 3.4, 33.4], $p<0.0001$; fully adjusted and removing deaths, OR 16.7 [95\% CI 3.9, 71.6], $p=0.0001$ ) and positive ZnT8 (unadjusted OR 4.0 [95\% CI 1.4 11.4], $p=0.01$; fully adjusted and removing deaths, OR 3.9 [95\% CI 1.2, 12.4], $p=0.02$; Table 3).

We analysed whether the presence of multiple autoantibodies might be significant (Table 4). The presence of one autoantibody compared with zero autoantibodies was associated with a significant increase in the odds of developing the adverse outcome (unadjusted OR 4.0 [95\% CI 3.0, 5.3], $p<0.0001$; adjusted OR 3.3 [95\% CI 2.4, 4.6], $p<0.0001$; fully adjusted and removing deaths, OR 3.4 [95\% CI 2.4, 4.7], $p<0.0001$ ), although all participants on baseline insulin were considered positive for IAA. The presence of two or more autoantibodies compared with zero autoantibodies was associated with even higher odds of the adverse outcome (unadjusted OR 12.4 [95\% CI 7.1, 21.6], $p<0.0001$; adjusted OR 9.2 [95\% CI 5.2, 16.5], $p<0.0001$; fully adjusted and removing deaths, OR 9.9 [95\% CI 5.4, 18.0], $p<0.0001$ ).

We also performed a sensitivity analysis excluding controls $(n=183)$ or cases $(n=16)$ not well-matched for age or BMI; these results were similar to those of the full sample.

\section{Discussion}

In this post hoc analysis of the ACCORD trial, we found that the presence of insulin deficiency (as measured by fasting $\mathrm{C}$ - 
Table 3 The presence of insulin deficiency or islet autoantibodies is associated with higher odds of both $\mathrm{SH}$ and failure to achieve $\mathrm{HbA}_{1 \mathrm{c}}<6.0$ (42 $\mathrm{mmol} / \mathrm{mol})$

\begin{tabular}{|c|c|c|c|}
\hline \multirow{2}{*}{$\begin{array}{l}\text { Variable by model } \\
\text { Fasting C-peptide }\end{array}$} & \multicolumn{2}{|c|}{$\begin{array}{l}\text { Odds of } \mathrm{SH} \text { accompanied by failure to } \\
\text { achieve } \mathrm{HbA}_{1 \mathrm{c}}<6.0 \% \text { ( } 42 \mathrm{mmol} / \mathrm{mol} \text {; } \\
\text { OR }[95 \% \mathrm{CI}])\end{array}$} & \multirow[t]{2}{*}{$p$ value } \\
\hline & Insulin sufficient & Insulin deficient & \\
\hline Model $1^{\mathrm{a}}$ & 1 (reference) & $35.6(14.2,89.6)$ & $<0.0001$ \\
\hline Model $2^{\mathrm{b}}$ & 1 (reference) & $24(9.4,61.5)$ & $<0.0001$ \\
\hline Model $3^{\mathrm{c}}$ & 1 (reference) & $23.2(9.0,59.5)$ & $<0.0001$ \\
\hline IAA/baseline insulin & Negative & Positive & \\
\hline Model $1^{\mathrm{a}}$ & 1 (reference) & $4.5(3.4,6.0)$ & $<0.0001$ \\
\hline Model $2^{\mathrm{b}}$ & 1 (reference) & $3.7(2.7,5.0)$ & $<0.0001$ \\
\hline Model $3^{\mathrm{c}}$ & 1 (reference) & $3.8(2.7,5.3)$ & $<0.0001$ \\
\hline GAD & Negative & Positive & \\
\hline Model $1^{\mathrm{a}}$ & 1 (reference) & $3.6(2.4,5.4)$ & $<0.0001$ \\
\hline Model $2^{\mathrm{b}}$ & 1 (reference) & $3.7(2.4,5.7)$ & $<0.0001$ \\
\hline Model $3^{\mathrm{c}}$ & 1 (reference) & $3.9(2.5,6.0)$ & $<0.0001$ \\
\hline IA2 & Negative & Positive & \\
\hline Model $1^{\mathrm{a}}$ & 1 (reference) & $10.6(3.4,33.4)$ & $<0.0001$ \\
\hline Model $2^{\mathrm{b}}$ & 1 (reference) & $11.9(3.3,42.3)$ & 0.0001 \\
\hline Model $3^{\mathrm{c}}$ & 1 (reference) & $16.7(3.9,71.6)$ & 0.0001 \\
\hline ZnT8 & Negative & Positive & \\
\hline Model $1^{\mathrm{a}}$ & 1 (reference) & $4.0(1.4,11.4)$ & 0.01 \\
\hline Model $2^{\mathrm{b}}$ & 1 (reference) & $3.5(1.2,10.4)$ & 0.03 \\
\hline Model $3^{\mathrm{c}}$ & 1 (reference) & $3.9(1.2,12.4)$ & 0.02 \\
\hline
\end{tabular}

${ }^{\text {a }}$ Model 1 is unadjusted

${ }^{\mathrm{b}}$ Model 2 is Model 1 with additional adjustment for age, BMI and diabetes duration

${ }^{\mathrm{c}}$ Model 3 is Model 2 re-analysed by removal of any cases who died $(n=$ $21)$ and their respective controls $(n=86)$

peptide and suggested by baseline insulin use) and islet autoantibodies were higher in participants who experienced $\mathrm{SH}$ and failed to achieve the intensive glycaemia target than in those who achieved an $\mathrm{HbA}_{1 \mathrm{c}}$ level of $<6.0 \%$ (42 mmol/ mol) without $\mathrm{SH}$. Our findings suggest that fasting C-peptide and islet autoantibodies (GAD, IA2, ZnT8) levels may serve as blood biomarkers to predict the risk of SH during intensification of type 2 diabetes treatment.

$\mathrm{SH}$ remains a devastating complication of diabetes treatment. In patients with type 2 diabetes, $\mathrm{SH}$ has been associated with an increased risk of mortality [3, 17] and emergency room visits [2]. Older age, diabetes duration, the presence of comorbidities, treatment intensification and insulin treatment have all been associated with an increased risk of hypoglycaemia [3-5, 18, 19]. Of particular importance are recent findings demonstrating an association between $\mathrm{SH}$ and insulin use [3-5], even in patients who do not achieve intensive glucose targets $[6,7,20]$. The current investigation presents blood biomarkers which are associated with $\mathrm{SH}$ and a failure to achieve an $\mathrm{HbA}_{1 \mathrm{c}}$ level of $<6.0(42 \mathrm{mmol} / \mathrm{mol})$ in the setting of intensive treatment, thereby providing potential tools to identify type 2 diabetes patients who can achieve lower $\mathrm{HbA}_{1 \mathrm{c}}$ targets without a significant risk of $\mathrm{SH}$.

The association between insulin deficiency and SH in type 2 diabetes has been described. With long-standing type 2 diabetes ( $>3$ years) and the presence of islet cell autoantibodies, patients with type 2 diabetes can develop insulin deficiency comparable to that of patients with type 1 diabetes [21], with similar hypoglycaemia rates [22]. It remains unclear whether the mechanism for this insulin deficiency arises from autoimmunity or progressive beta cell dysfunction. A subset (7$10 \%$ ) of patients with type 2 diabetes was positive for islet autoantibodies $[23,24]$. Such patients are said to have latent autoimmune diabetes of adults (LADA) [25]. The presence of LADA predicts the need for insulin treatment [23, 24, 26], but whether the presence of LADA might affect overall prognosis remains unclear. Since the study participants had clinically diagnosed type 2 diabetes and were retrospectively identified as positive for islet autoantibodies, our findings may represent the consequence of long-standing LADA and suggest a higher likelihood of SH and being unable to achieve a near-normal $\mathrm{HbA}_{1 \mathrm{c}}$ level.

The mechanism responsible for the increased risk of $\mathrm{SH}$ in insulin-deficient patients with type 2 diabetes is likely to be similar to that of patients with type 1 diabetes [27]. In the setting of insulin deficiency, one major mechanism for $\mathrm{SH}$

Table 4 The number of islet autoantibodies is associated with a higher odds of SH and failure to achieve $\mathrm{HbA}_{1 \mathrm{c}}<6.0(42 \mathrm{mmol} / \mathrm{mol})$

\begin{tabular}{|c|c|c|c|c|c|}
\hline \multirow[t]{2}{*}{ Model } & \multicolumn{5}{|c|}{ Odds of SH accompanied by failure to achieve $\mathrm{HbA}_{1 \mathrm{c}}<6.0 \%(42 \mathrm{mmol} / \mathrm{mol}$; OR $[95 \% \mathrm{CI}])$} \\
\hline & No autoantibodies & One autoantibody & $p$ value & $\geq$ Two autoantibodies & $p$ value \\
\hline Model $1^{\mathrm{a}}$ & 1 (reference) & $4.0(3.0,5.3)$ & $<0.0001$ & $12.4(7.1,21.6)$ & $<0.0001$ \\
\hline Model $2^{\mathrm{b}}$ & 1 (reference) & $3.3(2.4,4.6)$ & $<0.0001$ & $9.2(5.2,16.5)$ & $<0.0001$ \\
\hline Model $3^{\mathrm{c}}$ & 1 (reference) & $3.4(2.4,4.7)$ & $<0.0001$ & $9.9(5.4,18.0)$ & $<0.0001$ \\
\hline
\end{tabular}

${ }^{\text {a }}$ Model 1 is unadjusted

${ }^{\mathrm{b}}$ Model 2 is Model 1 with additional adjustment for age, BMI and diabetes duration

${ }^{\mathrm{c}}$ Model 3 is Model 2 re-analysed by removal of any cases who died $(n=21)$ and their respective controls $(n=86)$ 
risk is the loss of endogenous glucose-responsive insulin release, resulting in an inability to modulate endogenous insulin secretion given declining glucose levels and the presence of exogenous insulin which does not decrease as glucose levels fall [28]. Another potential mechanism is the impairment to hypoglycaemia-induced glucagon secretion. Consequently, these patients depend on hypoglycaemia-induced adrenaline and noradrenaline release to detect and respond to declining glucose levels [28]. Antecedent hypoglycaemia also alters the glucose threshold such that the catecholamine response is triggered at a lower plasma glucose, thereby increasing the risk of experiencing SH [29].

In terms of clinical relevance, this study demonstrates that blood biomarkers indicating insulin deficiency may predict the response of patients with type 2 diabetes to intensification of glycaemic treatment. The ADA released a consensus report suggesting that glycaemic goals may need to be liberalised in older patients with type 2 diabetes to reduce complications [30]. We propose that quantifying insulin deficiency in patients with type 2 diabetes may be important, as glycaemic goals may need to be liberalised for patients with type 2 diabetes and insulin deficiency irrespective of age, particularly if the risks of SH may outweigh the benefits of intensive glycaemic control. Future studies will be necessary to determine whether individualising glycaemic goals based on blood biomarkers of insulin deficiency or islet autoantibodies will improve outcomes for patients with type 2 diabetes.

This study had several strengths. Baseline fasting Cpeptide and islet autoantibody levels were measured by standardised assays. Intensive diabetes treatment was performed using a standard protocol as per the ACCORD study. Outcomes were measured prospectively using protocols with a standardised definition of hypoglycaemia, assessment of $\mathrm{HbA}_{1 \mathrm{c}}$ levels and centralised laboratory measurement. The results remained highly significant even after adjustment for age and diabetes duration. Several study limitations exist. Given the nested case-control design, re-analysing this cohort by defining cases as those participants who failed to achieve a target $\mathrm{HbA}_{1 \mathrm{c}}$ level of $<6.0 \%$ (42 $\left.\mathrm{mmol} / \mathrm{mol}\right)$ regardless of $\mathrm{SH}$ or by defining cases as those participants who had SH regardless of the $\mathrm{HbA}_{1 \mathrm{c}}$ level achieved would provide additional insights; however, this could not be performed in the present analysis as biomarker data was not measured in the complete ACCORD cohort. Since blood biomarkers were measured in only a selected population from the ACCORD trial rather than all participants, only a small number of positive islet autoantibodies (excluding IAA which we designated as positive in the setting of insulin use) were discovered. Consequently, the generalisability of these significant findings remains limited and requires replication in a larger cohort. We acknowledge that patients with LADA or type 1 diabetes may have been inadvertently recruited for ACCORD. However, given the clinical screening prior to recruitment, these patients, even if inadvertently recruited, have a phenotypical presentation similar to patients with clinical type 2 diabetes. The use of insulin may confound our observations, as the cases may represent a group of patients with difficult-to-control type 2 diabetes, and the higher rate of insulin use may increase the frequency of hypoglycaemic events. The fasting C-peptide measurement has some constraints. Fasting C-peptide and glucose levels are simplified measures of insulin deficiency. Insulin treatment may lower fasting C-peptide levels by suppressing endogenous glucose production. Fasting glucose levels may influence fasting C-peptide levels, although the mean fasting glucose level was not low (mean $\pm \mathrm{SD}$; cases $9.4 \pm 4.1 \mathrm{mmol} /$ 1; controls $9.8 \pm 2.7 \mathrm{mmol} / \mathrm{l}$ ). As a consequence of the post hoc analysis, we acknowledge that the potential causal and associative natures of the blood biomarkers cannot be distinguished.

\section{Conclusions}

In the ACCORD study, baseline measures of C-peptide and islet autoantibodies are associated with $\mathrm{SH}$ during intensification of type 2 diabetes treatment. Since a major goal of type 2 diabetes treatment is to minimise patient risk of microvascular complications without increasing the therapy burden, these blood biomarkers may prove useful in the individualisation of type 2 diabetes treatment.

Acknowledgements The authors thank the staff and participants of the ACCORD study for their important contributions.

Some of these data were presented at the ADA 74th Scientific Sessions in 2014 [31].

Funding We acknowledge funding from the National Institutes of Health (1R01DK095360-01) for this study. The ACCORD study was supported by contracts from the National Heart Lung and Blood Institute (N01-HC-95178, N01-HC-95179, N01-HC-95180,N01-HC95181, N01-HC-95182, N01-HC-95183, N01-HC-95184, IAA-Y1-HC9035 and IAA-Y1-HC-1010) and by other components of the National Institutes of Health (including the NIDDK, the National Institute on Aging, and the National Eye Institute), the Centers for Disease Control and Prevention and General Clinical Research Centers. The following companies provided study medications, equipment or supplies: Abbott Laboratories, Amylin Pharmaceutical, AstraZeneca, Bayer HealthCare, Closer Healthcare, GlaxoSmithKline, King Pharmaceuticals, Merck, Novartis, Novo Nordisk, Omron Healthcare, Sanofi-Aventis and Schering-Plough.

Duality of interest The authors declare that there is no duality of interest associated with this manuscript.

Contribution statement LSC, MEM and ERS designed the study; SMM provided essential data for analysis; LSC, HC, MEM and ERS analysed the data; LSC, HC, MEM, SMM and ERS wrote the manuscript; and LSC, HC, MEM, SMM and ERS contributed to discussions and reviewed and edited the manuscript. LSC has primary responsibility for the final content. All authors have read and approved the final manuscript. 


\section{References}

1. Bonds DE, Kurashige EM, Bergenstal R et al (2007) Severe hypoglycemia monitoring and risk management procedures in the Action to Control Cardiovascular Risk in Diabetes (ACCORD) Trial. Am J Cardiol 99:S80-S89

2. Leese GP, Wang J, Broomhall J et al (2003) Frequency of severe hypoglycemia requiring emergency treatment in type 1 and type 2 diabetes: a population-based study of health service resource use. Diabetes Care 26:1176-1180

3. Zoungas S, Patel A, Chalmers J et al (2010) Severe hypoglycemia and risks of vascular events and death. N Engl J Med 363:1410-1418

4. Turnbull FM, Abraira C, Anderson RJ et al (2009) Intensive glucose control and macrovascular outcomes in type 2 diabetes. Diabetologia 52:2288-2298

5. Miller ME, Bonds DE, Gerstein HC et al (2010) The effects of baseline characteristics, glycaemia treatment approach, and glycated haemoglobin concentration on the risk of severe hypoglycaemia: post hoc epidemiological analysis of the ACCORD study. BMJ 340:b5444

6. Lipska KJ, Warton EM, Huang ES et al (2013) $\mathrm{HbA}_{1 \mathrm{c}}$ and risk of severe hypoglycemia in type 2 diabetes: the diabetes and aging study. Diabetes Care 36:3535-3542

7. McCoy RG, Van Houten HK, Ziegenfuss JY, Shah ND, Wermers RA, Smith SA (2012) Increased mortality of patients with diabetes reporting severe hypoglycemia. Diabetes Care 35:1897-1901

8. Siegelaar SE, Holleman F, Hoekstra JBL, DeVries JH (2010) Glucose variability; does it matter? Endocr Rev 31:171-182

9. Buse JB, Bigger JT, Byington RP et al (2007) Action to Control Cardiovascular Risk in Diabetes (ACCORD) trial: design and methods. Am J Cardiol 99:21i-33i

10. Bergstralh EJ, Kosanke J. (2003) Locally written SAS macros. Available from www.mayo.edu/research/departments-divisions/ department-health-sciences-research/division-biomedical-statisticsinformatics/software/locally-written-sas-macros. Accessed July 2014

11. Bergstralh EJ, Kosanke J. (2014) Locally written SAS macros. Available from www.mayo.edu/research/departments-divisions/ department-health-sciences-research/division-biomedical-statisticsinformatics/software/locally-written-sas-macros. Accessed July 2014

12. Bergstralh EJ, Kosanke JL, Jacobsen SJ (1996) Software for optimal matching in observational studies. Epidemiol (Camb Mass) 7:331-332

13. Berger B, Stenstrom G, Sundkvist G (2000) Random C-peptide in the classification of diabetes. Scand J Clin Lab Investig 60:687-693

14. Jones AG, Hattersley AT (2013) The clinical utility of C-peptide measurement in the care of patients with diabetes. Diabet Med 30:803-817

15. Bonifacio E, Yu L, Williams AK et al (2010) Harmonization of glutamic acid decarboxylase and islet antigen-2 autoantibody assays for National Institute of Diabetes and Digestive and Kidney Diseases Consortia. J Clin Endocrinol Metab 95:3360-3367

16. Schernthaner G (1993) Immunogenicity and allergenic potential of animal and human insulins. Diabetes Care 16:155-165

17. Bonds DE, Miller ME, Bergenstal RM et al (2010) The association between symptomatic, severe hypoglycaemia and mortality in type 2 diabetes: retrospective epidemiological analysis of the ACCORD study. BMJ 340:b4909

18. Alsahli M, Gerich JE (2013) Hypoglycemia. Endocrinol Metab Clin N Am 42:657-676

19. Amiel SA, Dixon T, Mann R, Jameson K (2008) Hypoglycaemia in type 2 diabetes. Diabet Med 25:245-254

20. Mitchell BD, Vietri J, Zagar A, Curtis B, Reaney M (2013) Hypoglycaemic events in patients with type 2 diabetes in the United Kingdom: associations with patient-reported outcomes and self-reported $\mathrm{HbA}_{1 \mathrm{c}}$. BMC Endocr Disord 13:59

21. Gottsater A, Landin-Olsson M, Fernlund P, Lernmark A, Sundkvist G (1993) Beta-cell function in relation to islet cell antibodies during the first $3 \mathrm{yr}$ after clinical diagnosis of diabetes in type II diabetic patients. Diabetes Care 16:902-910

22. Group UHS (2007) Risk of hypoglycaemia in types 1 and 2 diabetes: effects of treatment modalities and their duration. Diabetologia 50: $1140-1147$

23. Irvine WJ, Gray RS, McCallum CJ, Duncan LJP (1977) Clinical and pathogenic significance of pancreatic-islet-cell antibodies in diabetics treated with oral hypoglycaemic agents. Lancet 1:1025-1027

24. Hawa MI, Buchan AP, Ola T et al (2014) LADA and CARDS: a prospective study of clinical outcome in established adult-onset autoimmune diabetes. Diabetes Care 37:1643-1649

25. Zimmet PZ (1995) The pathogenesis and prevention of diabetes in adults. Genes, autoimmunity, and demography. Diabetes Care 18: 1050-1064

26. Fourlanos S, Dotta F, Greenbaum CJ et al (2005) Latent autoimmune diabetes in adults (LADA) should be less latent. Diabetologia 48: 2206-2212

27. Cryer PE (2004) Diverse causes of hypoglycemia-associated autonomic failure in diabetes. N Engl J Med 350:2272-2279

28. Cryer PE, Davis SN, Shamoon H (2003) Hypoglycemia in diabetes. Diabetes Care 26:1902-1912

29. Segel SA, Paramore DS, Cryer PE (2002) Hypoglycemia-associated autonomic failure in advanced type 2 diabetes. Diabetes 51:724-733

30. Kirkman MS, Briscoe VJ, Clark N et al (2012) Diabetes in older adults. Diabetes Care 35:2650-2664

31. Chow LS CH, Miller M, Marcovina S, Seaquist E (2014) Biomarkers associated with severe hypoglycemia and failure to achieve good glycemic control in T2DM: the ACCORD study. Diabetes 63(Supp11):A45, Abstract 